\title{
Stratospheric benzene and hydrocarbon aerosols detected in Saturn's auroral regions
}

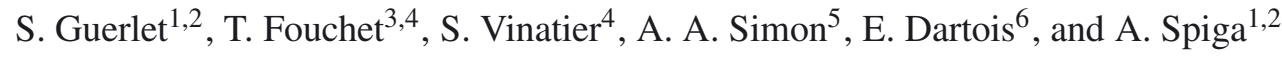 \\ 1 Sorbonne Universités, UPMC Paris 06, UMR 8539, LMD, 75005 Paris, France \\ e-mail: sandrine.guerlet@lmd.jussieu.fr \\ 2 CNRS, Laboratoire de Météorologie Dynamique, IPSL, UMR 8539, 4 place Jussieu, 75005 Paris, France \\ 3 Sorbonne Universités, UPMC Paris 06, UMR 8109, LESIA 75005 Paris, France \\ ${ }^{4}$ LESIA, Observatoire de Paris, CNRS, UPMC, Université Paris-Diderot, 5 place Jules Janssen, 92195 Meudon, France \\ 5 NASA Goddard Space Flight Center, 8800 Greenbelt Road, Greenbelt, MD 20771, USA \\ ${ }^{6}$ CNRS-INSU, Institut d'Astrophysique Spatiale, UMR 8617, 91405 Orsay, France
}

Received 4 August 2014 / Accepted 8 June 2015

\begin{abstract}
Context. Saturn's polar upper atmosphere exhibits significant auroral activity; however, its impact on stratospheric chemistry (i.e. the production of benzene and heavier hydrocarbons) and thermal structure remains poorly documented.

Aims. We aim to bring new constraints on the benzene distribution in Saturn's stratosphere, to characterize polar aerosols (their vertical distribution, composition, thermal infrared optical properties), and to quantify the aerosols' radiative impact on the thermal structure.

Methods. Infrared spectra acquired by the Composite Infrared Spectrometer (CIRS) on board Cassini in limb viewing geometry are analysed to derive benzene column abundances and aerosol opacity profiles over the 3 to 0.1 mbar pressure range. The spectral dependency of the haze opacity is assessed in the ranges 680-900 and 1360-1440 $\mathrm{cm}^{-1}$. Then, a radiative climate model is used to compute equilibrium temperature profiles, with and without haze, given the haze properties derived from CIRS measurements.

Results. On Saturn's auroral region $\left(80^{\circ} \mathrm{S}\right)$, benzene is found to be slightly enhanced compared to its equatorial and mid-latitude values. This contrasts with the Moses \& Greathouse (2005, J. Geophys. Res., 110, 9007) photochemical model, which predicts a benzene abundance 50 times lower at $80^{\circ} \mathrm{S}$ than at the equator. This advocates for the inclusion of ion-related reactions in Saturn's chemical models. The polar stratosphere is also enriched in aerosols, with spectral signatures consistent with vibration modes assigned to aromatic and aliphatic hydrocarbons, and presenting similarities with the signatures observed in Titan's stratosphere. The aerosol mass loading at $80^{\circ} \mathrm{S}$ is estimated to be $1-4 \times 10^{-5} \mathrm{~g} \mathrm{~cm}^{-2}$, an order of magnitude less than on Jupiter, which is consistent with the order of magnitude weaker auroral power at Saturn. We estimate that this polar haze warms the middle stratosphere by $6 \mathrm{~K}$ in summer and cools the upper stratosphere by $5 \mathrm{~K}$ in winter. Hence, aerosols linked with auroral activity can partly account for the warm polar hood observed in Saturn's summer stratosphere.
\end{abstract}

Key words. planets and satellites: gaseous planets - planets and satellites: atmospheres - planets and satellites: composition planets and satellites: aurorae

\section{Introduction}

Saturn's polar regions are characterized by permanent dark polar caps in the ultraviolet, whose latitudinal extent coincides with that of auroral emissions (Gérard et al. 1995). These dark regions are generally attributed to the presence of polar stratospheric hazes (West et al. 1983; Karkoschka \& Tomasko 1993), possibly produced by the precipitation of energetic auroral electrons (Pryor \& Hord 1991). Previous observations, in particular from the Hubble Space Telescope (HST), have allowed others (Karkoschka \& Tomasko 2005; Pérez-Hoyos et al. 2005) to derive the size, optical depth, and refractive indexes of stratospheric aerosol particles in the visible and UV. Both studies reveal that aerosol particles exhibit different properties poleward of $\sim 70^{\circ}$, being much more absorbent at UV wavelengths, with an imaginary refractive index one to two orders of magnitude larger than at mid-latitudes. However, these observations alone cannot constrain the chemical nature of these aerosols or their radiative impact on the stratospheric temperature.
In parallel, the benzene $\left(\mathrm{C}_{6} \mathrm{H}_{6}\right)$ column density has been measured in the stratospheres of Jupiter and Saturn (disk average) using the Infrared Space Observatory (Bézard et al. 2001). In the case of Jupiter's polar atmosphere, benzene has been proposed as a precursor to the formation of more complex hydrocarbons, such as polyacetylenes and multi-ring compounds, which could condense to form polar aerosols (Wong et al. 2000, 2003; Friedson et al. 2002). This formation scenario, in which ion chemistry plays a key role, could also apply to Saturn, but both observational and modelling constraints to test this scenario are missing. In this context, the goal of our study is to search for tracers of ion chemistry (benzene and aerosols) in Saturn's neutral stratosphere.

Here we report on the first measurement of benzene column abundance in Saturn's polar stratosphere, together with the first detection of spectral signatures of the polar haze in the thermal infrared, based on limb measurements from the Composite Infrared Spectrometer (CIRS) on board Cassini. These measurements allow us to provide new constraints on the benzene 
Table 1. Characteristics of the limb datasets analysed in this study.

\begin{tabular}{ccccc}
\hline \hline Latitude & Date & $L_{\mathrm{S}}$ & Spectral resolution & Number of co-added spectra \\
\hline $40^{\circ} \mathrm{N}$ & 23 Aug. 2011 & $25^{\circ}$ & $0.5 \mathrm{~cm}^{-1}$ & 120 \\
$0^{\circ}$ & 3 Jan. 2008 & $340^{\circ}$ & $0.5 \mathrm{~cm}^{-1}$ & 57 \\
$35^{\circ} \mathrm{S}$ & 4 Jan. 2012 & $30^{\circ}$ & $0.5 \mathrm{~cm}^{-1}$ & 82 \\
$80^{\circ} \mathrm{S}$ & 28 Jun. 2007 & $333^{\circ}$ & $1.5 \mathrm{~cm}^{-1}$ & 100 \\
\hline
\end{tabular}

Notes. $L_{\mathrm{S}}$ refers to solar longitude $\left(0^{\circ}\right.$ at northern spring equinox).

chemistry and on the nature and origin of the polar haze. In addition, by combining the haze optical properties in the visible and thermal infrared with their sizes and opacity vertical profiles, we then evaluate the radiative impact of Saturn's polar stratospheric haze on the thermal structure.

\section{Cassini/CIRS limb observations}

CIRS is a Fourier transform spectrometer comprising three focal planes, covering the regions $10-600 \mathrm{~cm}^{-1}$ (FP1), 570$1125 \mathrm{~cm}^{-1}$ (FP3), and 1025-1495 $\mathrm{cm}^{-1}$ (FP4) with a spectral resolution tunable between 0.5 and $15 \mathrm{~cm}^{-1}$ for apodized spectra $\left(0.25\right.$ to $7.5 \mathrm{~cm}^{-1}$ for unapodized spectra). A detailed description of CIRS technical characteristics and observing modes can be found in Flasar et al. (2004). Here we report on the analysis of unapodized spectra acquired in limb viewing geometry by FP3 and FP4. Each of these two focal planes consists of a linear array of ten square detectors with an individual projected field of view that is approximately Saturn's atmospheric scale height $(\sim 60 \mathrm{~km})$. The two detector arrays are set perpendicular to the limb of the planet, pointing to a given latitude. Data are typically acquired for $20 \mathrm{~min}$ (if the lower spectral resolution is used) before shifting the detector arrays along their $Z$-axis by two-thirds the arrays' length, in order to increase the vertical coverage ( $\sim 1000 \mathrm{~km}$ by combining the two positions). Hence, limb observations by CIRS achieve both a good vertical extent and resolution.

In previous studies, limb data acquired in 2005-2010 (mostly at a $7.5 \mathrm{~cm}^{-1}$ resolution) have been used to retrieve vertical profiles of the stratospheric temperature and of the abundance of five hydrocarbons $\left(\mathrm{C}_{2} \mathrm{H}_{6}, \mathrm{C}_{2} \mathrm{H}_{2}, \mathrm{C}_{3} \mathrm{H}_{8}, \mathrm{CH}_{3} \mathrm{C}_{2} \mathrm{H}, \mathrm{C}_{4} \mathrm{H}_{2}\right)$, the main by-products of methane photolysis (Fouchet et al. 2008; Guerlet et al. 2009, 2010). In these studies, even if thermal emission by aerosols was neglected, a satisfactory agreement between the synthetic and the observed spectra was achieved for the vast majority of the dataset.

Here we focus on the analysis of four limb datasets acquired at a spectral resolution of $0.5 \mathrm{~cm}^{-1}$ and $1.5 \mathrm{~cm}^{-1}$, at latitudes $40^{\circ} \mathrm{N}$, the equator, $35^{\circ} \mathrm{S}$, and $80^{\circ} \mathrm{S}$ (within the polar cap). The characteristics of these limb data (date, latitude, spectral resolution, and number of individual spectra co-added) are summarized in Table 1. At these spectral resolutions, in addition to the five aforementioned hydrocarbons detected at a $7.5 \mathrm{~cm}^{-1}$ resolution, the narrow signature of benzene appears at $674 \mathrm{~cm}^{-1}$, together with the neighbouring $\mathrm{CO}_{2}$ band at $667 \mathrm{~cm}^{-1}$. While the $\mathrm{CO}_{2}$ band is detected at all four latitudes, benzene is only clearly detected at $80^{\circ} \mathrm{S}$, and marginally at $40^{\circ} \mathrm{N}$. In addition, at $80^{\circ} \mathrm{S}$, in contrast to mid- and low-latitude data, several unknown residual spectral features could not be reproduced by the nominal radiative transfer model used by Guerlet et al. (2009). These features are suggestive of polar aerosols, which is further investigated below. The retrieval of atmospheric profiles (temperature, trace gaseous species, and aerosol opacities) is described in the following section, along with examples of CIRS spectra exhibiting benzene and aerosol signatures.

\section{Data analysis}

\subsection{Retrieval of the temperature and main hydrocarbon profiles}

In the following, we employ the forward radiative transfer model coupled to the Bayesian inversion method already described in Guerlet et al. (2009); we only recall here their main characteristics. The first step consists in retrieving a vertical temperature profile from the combined analysis of the $v_{4}$ methane band (1200-1370 $\left.\mathrm{cm}^{-1}\right)$, which probes the middle and upper stratosphere, and of the $\mathrm{H}_{2}-\mathrm{H}_{2}$ and $\mathrm{H}_{2}-\mathrm{He}$ collision-induced emission $\left(590-660 \mathrm{~cm}^{-1}\right)$, probing the lower stratosphere. This requires an assumption on the methane abundance and the $\mathrm{He} / \mathrm{H}_{2}$ ratio. Both are assumed to be homogeneously distributed in Saturn's stratosphere. The deep mixing ratio of $\mathrm{CH}_{4}$ is set to 0.0045 (Flasar et al. 2005) and then decreases with height following the Moses \& Greathouse (2005) modelled vertical profile. The $\mathrm{H}_{2}$ and $\mathrm{He}$ mole fractions are set to 0.86 and 0.1355 , respectively, as obtained by Conrath \& Gautier (2000). Temperature profiles are retrieved through an iterative procedure that minimizes a two-term cost function, with one term minimizing the difference between synthetic spectra and the measured ones, and a regularization term ensuring that the retrieved profiles do not exhibit non-physical oscillating patterns and do not depart too much from a prior profile. For each limb dataset, multiple retrievals are performed starting from "cold" and "warm" prior temperature profiles and for different regularization parameters, in order to carefully assess the robustness of our method and select the best solution profile.

A simultaneous analysis of CIRS limb spectra acquired at different tangent altitudes (typically ten) allows us to constrain the temperature profile between 20 mbar and a few $\mu$ bar. Figure 1 compares the temperature profiles retrieved at the four latitudes considered in this paper. In the lower stratosphere (1-20 mbar), the temperature is the warmest at $80^{\circ} \mathrm{S}$, which is expected given the observing conditions at that time (during the summer polar day). At the 2 mbar pressure level, the temperature ranges between $138 \mathrm{~K}\left(35^{\circ} \mathrm{S}\right.$, autumn $)$ and $159 \mathrm{~K}\left(80^{\circ} \mathrm{S}\right.$, summer $)$. Warmer temperatures will favour the detection of benzene and aerosols, as discussed in Sect. 3.2. We note that the $40^{\circ} \mathrm{N}$ measurement was performed at the latitude and time of the powerful storm observed in late 2010 and 2011 , but $\sim 140^{\circ}$ away (in longitude) from the warm stratospheric "beacon" (Fletcher et al. 2012).

In addition, once the temperature is set, vertical profiles of the volume mixing ratio of several minor species can be retrieved from the analysis of their emission bands with a similar retrieval algorithm. In a given spectral interval, our strategy is to start from the retrieval of the species contributing the most to the 
S. Guerlet et al.: Benzene and hydrocarbon aerosols in Saturn's polar stratosphere

Table 2. Spectral ranges considered for the retrieval of hydrocarbon profiles and a list of other opacity sources considered in each spectral windows.

\begin{tabular}{ccc}
\hline \hline Spectral interval & Retrieved species and vertical extent & Other species considered \\
\hline $780-860 \mathrm{~cm}^{-1}$ & $\mathrm{C}_{2} \mathrm{H}_{6}(5 \mathrm{mbar}-1 \mu \mathrm{bar})$ & $\mathrm{C}_{2} \mathrm{H}_{2}$ \\
$700-760 \mathrm{~cm}^{-1}$ & $\mathrm{C}_{2} \mathrm{H}_{2}(5 \mathrm{mbar}-1 \mu$ bar $)$ and $\mathrm{C}_{3} \mathrm{H}_{8}(5-0.5 \mathrm{mbar})$ & $\mathrm{C}_{2} \mathrm{H}_{6}$ \\
$620-640 \mathrm{~cm}^{-1}$ & $\mathrm{CH}_{3} \mathrm{C}_{2} \mathrm{H}(5-0.01 \mathrm{mbar})$ and $\mathrm{C}_{4} \mathrm{H}_{2}(3-0.01 \mathrm{mbar})$ & $\mathrm{C}_{2} \mathrm{H}_{2}$ \\
$660-680 \mathrm{~cm}^{-1}$ & $\mathrm{CO}_{2}(3-0.1 \mathrm{mbar}), \mathrm{C}_{6} \mathrm{H}_{6}(3-0.2 \mathrm{mbar}$, col. abundance $)$ & $\mathrm{C}_{2} \mathrm{H}_{2}$ \\
\hline
\end{tabular}

Notes. The pressure range of maximum sensitivity to hydrocarbons is also given.

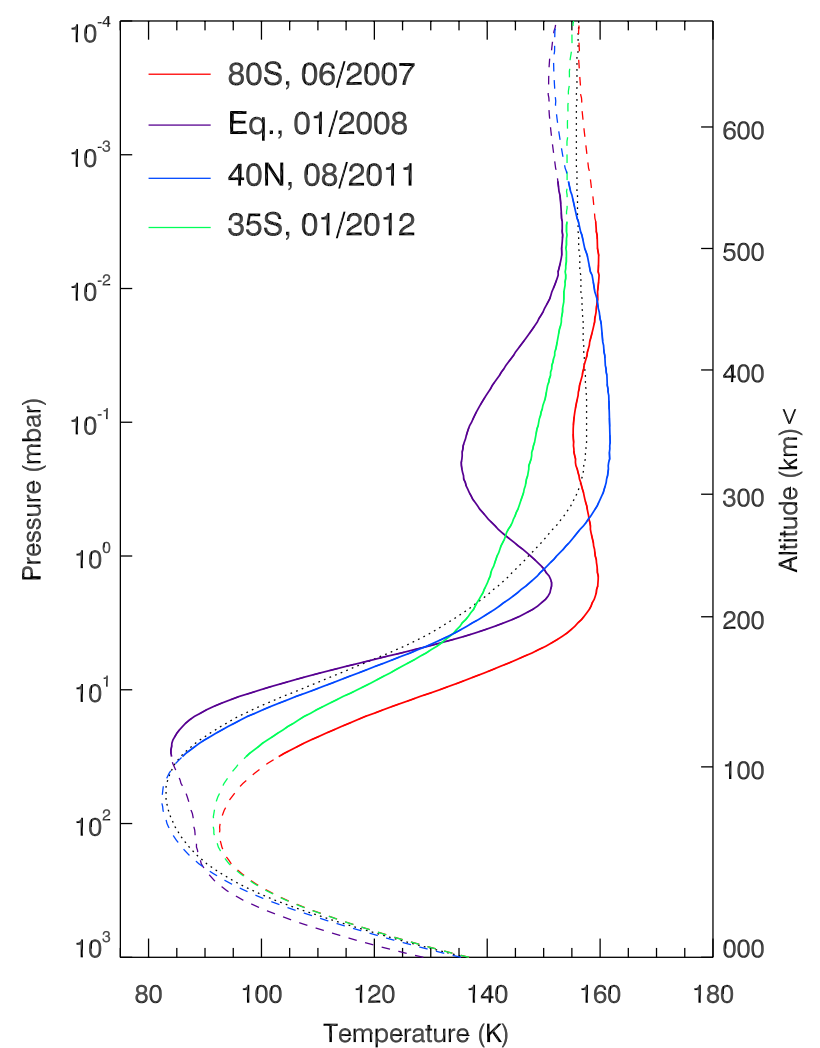

Fig. 1. Temperature profiles retrieved from Cassini/CIRS limb data at $80^{\circ} \mathrm{S}$ (in red), $35^{\circ} \mathrm{S}$ (green), the equator (purple), and $40^{\circ} \mathrm{N}$ (blue). Solid lines highlight the pressure range of maximum sensitivity to the temperature. The a priori profile is shown as the black dotted line.

opacity, or to retrieve simultaneously the profiles of two species if their emission bands are close. Table 2 summarizes the retrieved species for each spectral interval considered, along with the pressure range of maximum sensitivity to each hydrocarbon. The wider vertical sensitivity is achieved for the two dominant by-products of methane photochemistry, ethane and acetylene, whose signatures are detected on spectra acquired at 7-8 different tangent altitudes. Their mixing ratio profiles are constrained between 5 mbar and a few $\mu$ bar.

Details on the error budget and sensitivity tests can be found in Guerlet et al. $(2009,2010)$ for the five main hydrocarbons, and in the following section for benzene.

\subsection{Retrieval of the benzene abundance profile}

Here we focus on the $660-680 \mathrm{~cm}^{-1}$ region where benzene is detected for the first time in Cassini/CIRS spectra; it is detected clearly at $80^{\circ} \mathrm{S}$ at two different tangent altitudes (2 to $3 \sigma$ above the noise level) and more marginally at $40^{\circ} \mathrm{N}$ ( 1 to $2 \sigma$ above the noise level). Whether the stronger benzene detection at $80^{\circ} \mathrm{S}$ is
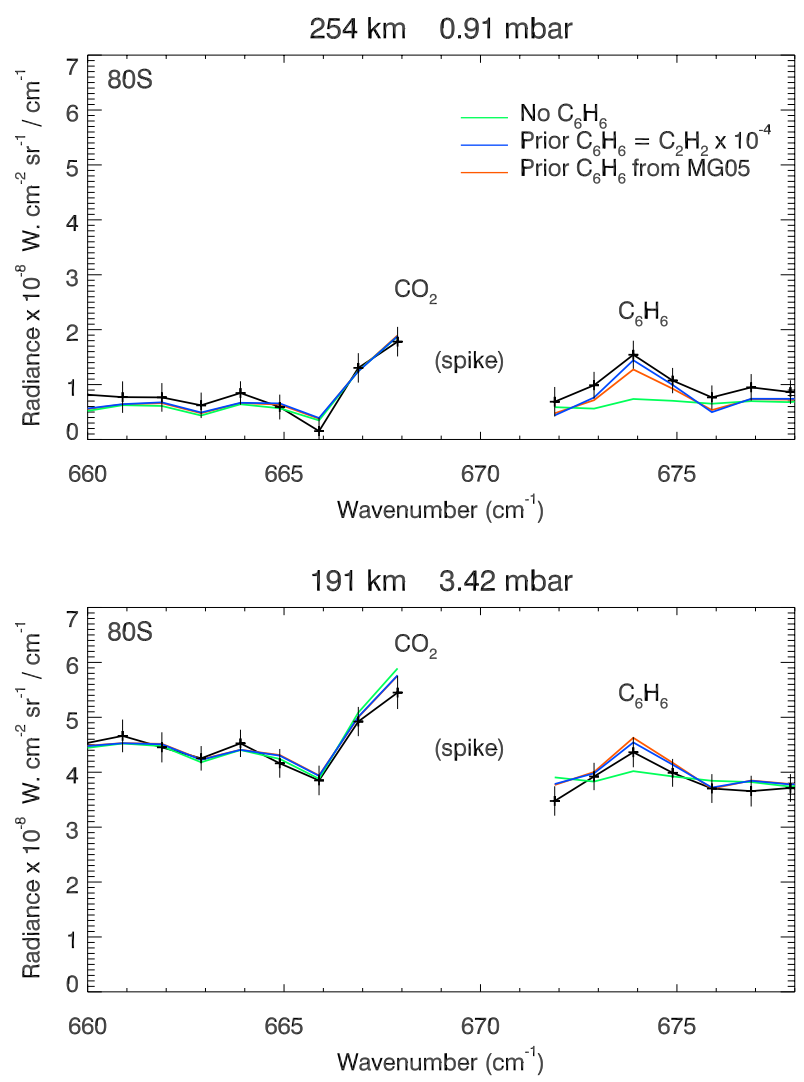

Fig. 2. Cassini/CIRS limb spectra acquired at two different tangent pressure levels at $80^{\circ} \mathrm{S}$ (in black, with $1 \sigma$ error bars). Best-fit synthetic spectra are overplotted, corresponding to the retrieval of the $\mathrm{CO}_{2}$ mixing ratio alone (in green, no benzene opacity) or to the simultaneous retrieval of $\mathrm{CO}_{2}$ and $\mathrm{C}_{6} \mathrm{H}_{6}$ mixing ratios (in blue and red, assuming different $\mathrm{C}_{6} \mathrm{H}_{6}$ vertical distributions shown in Fig. 4, same colour code). Wavenumbers contaminated by an electrical noise spike are omitted in the analysis.

driven by warmer stratospheric temperatures and/or by a higher benzene abundance needs to be assessed by the formal retrieval of the benzene profile at $80^{\circ} \mathrm{S}$ and $40^{\circ} \mathrm{N}$, and its upper limit at other latitudes.

Figures 2 and 3 present examples of CIRS spectra at $80^{\circ} \mathrm{S}$ and $40^{\circ} \mathrm{N}$, along with best-fit spectra corresponding to the simultaneous retrieval of the $\mathrm{C}_{6} \mathrm{H}_{6}$ and $\mathrm{CO}_{2}$ mixing ratio profiles. A test case of a $\mathrm{CO}_{2}$ retrieval where the $\mathrm{C}_{6} \mathrm{H}_{6}$ opacity is omitted is also shown to highlight the $\mathrm{C}_{6} \mathrm{H}_{6}$ detection. The corresponding $\mathrm{C}_{6} \mathrm{H}_{6}$ abundance profiles retrieved at $80^{\circ} \mathrm{S}$ are presented in Fig. 4. The sensitivity of $\mathrm{C}_{6} \mathrm{H}_{6}$ retrieval to the choice of the prior profile is tested by starting from prior $\mathrm{C}_{6} \mathrm{H}_{6}$ profiles with very different vertical distributions: one based on the Moses \& Greathouse (2005) photochemical model (hereafter referred to as MG05, which does not include ion chemistry and decreases 

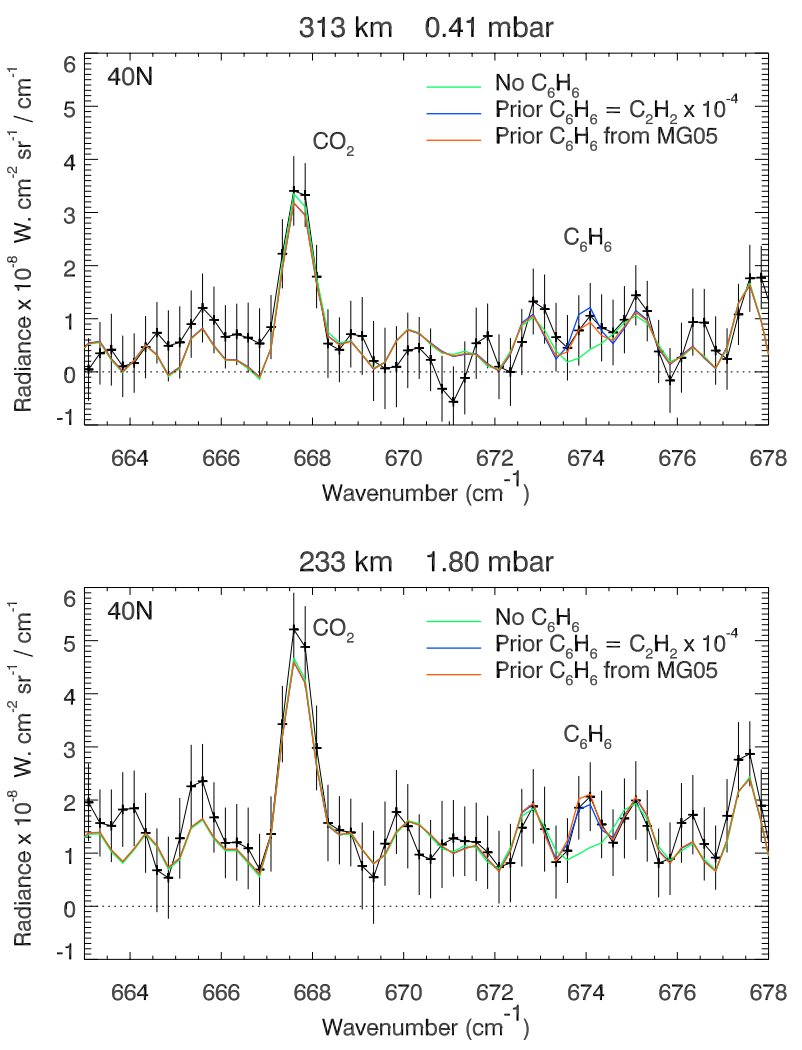

Fig. 3. Similar to Fig. 2, but for latitude $40^{\circ} \mathrm{N}$ and acquired at a higher spectral resolution, see Table 1.

above the 1 mbar level) and the other equal to the retrieved $\mathrm{C}_{2} \mathrm{H}_{2}$ profile divided by 10,000 (which increases with height, see Fig. 4). The two retrieved $\mathrm{C}_{6} \mathrm{H}_{6}$ profiles, although very different, correspond to similar fits of the data (see Fig. 2), showing that we cannot constrain the $\mathrm{C}_{6} \mathrm{H}_{6}$ vertical distribution. However, the two $\mathrm{C}_{6} \mathrm{H}_{6}$ column densities integrated between 3 mbar and 0.2 mbar (corresponding to the range of maximum sensitivity to benzene) agree within the error bars. Hence the retrieved partial column is considered a more robust quantity than the volume mixing ratio profiles.

We derive a partial column of $4.1 \pm 1.0 \times 10^{13} \mathrm{~cm}^{-2}$ at $80^{\circ} \mathrm{S}$ and $2.3 \pm 0.5 \times 10^{13}$ or $3.3 \pm 0.6 \times 10^{13} \mathrm{~cm}^{-2}$ at $40^{\circ} \mathrm{N}$, depending on the prior profile used. The error on the benzene integrated column abundance includes the propagation of the noise in the data, a smoothing error pertaining to the inverse method as well as the propagation of the error on the temperature profile $(0.5-1 \mathrm{~K}$ in this pressure range). Spectroscopic uncertainties on $\mathrm{C}_{6} \mathrm{H}_{6}$ would yield a systematic error at all latitudes and are not considered in this error budget, as our goal is to study meridional variations.

For comparison, using ISO measurements, Bézard et al. (2001) derived a disk-average column density of $4.7 \pm 0.9 \times$ $10^{13} \mathrm{~cm}^{-2}$ above the $10 \mathrm{mbar}$ pressure level. Our retrieved profiles at $40^{\circ} \mathrm{N}$ and $80^{\circ} \mathrm{S}$, extrapolated to this pressure level, yield columns of 3.5 and $5.7 \pm 0.9 \times 10^{13} \mathrm{~cm}^{-2}$, respectively, bracketing the ISO measurements.

At other latitudes, we determine an upper limit for the $\mathrm{C}_{6} \mathrm{H}_{6}$ column density by setting its mixing ratio profile to the above-mentioned prior profiles multiplied by a scaling factor. The upper limit for $\mathrm{C}_{6} \mathrm{H}_{6}$ corresponds to the column density for which the benzene emission band would be detected above the $1 \sigma$ noise level. In order to illustrate the sensitivity of the limb spectra to the benzene abundance, Figs. 5 and 6 compare CIRS spectra at $35^{\circ} \mathrm{S}$ and at the equator with synthetic spectra

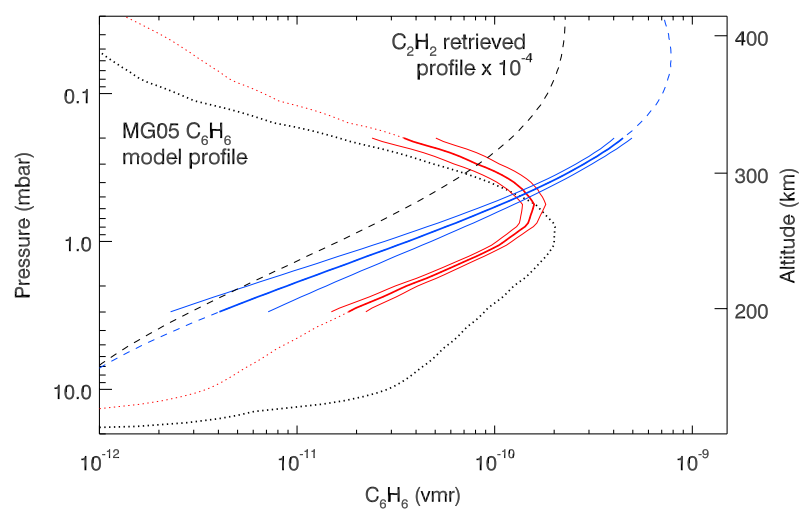

Fig. 4. $\mathrm{C}_{6} \mathrm{H}_{6}$ prior profiles (in black) and retrieved profiles at $80^{\circ} \mathrm{S}$ (red and blue), with thick solid lines highlighting the vertical extent of sensitivity to $\mathrm{C}_{6} \mathrm{H}_{6}$ (3-0.2 mbar). The thin solid lines highlight the error envelope that includes the propagation of the noise in the data and a smoothing error.
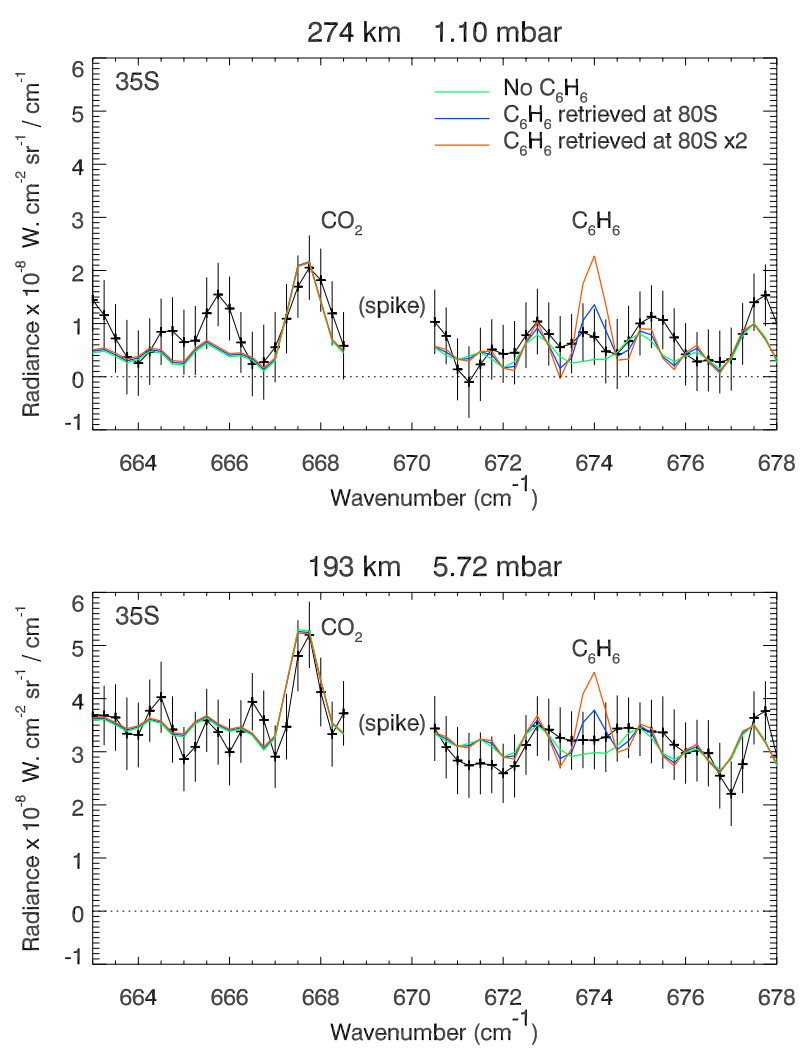

Fig. 5. Cassini/CIRS limb spectra acquired at two different tangent pressure levels at $35^{\circ} \mathrm{S}$. Synthetic spectra corresponding to the retrieval of the $\mathrm{CO}_{2}$ mixing ratio are overplotted, either assuming no benzene opacity (in green) or setting the benzene vertical profile to that retrieved at $80^{\circ} \mathrm{S}$ (in blue), or to that at $80^{\circ} \mathrm{S}$ multiplied by two (in red).

for which the benzene abundance was set to that retrieved at $80^{\circ} \mathrm{S}$, and at $80^{\circ} \mathrm{S}$ multiplied by two. While the first test case cannot be completely ruled out (synthetic spectra are compatible with observations within the $2 \sigma$ error bars), a scenario with twice that amount of $\mathrm{C}_{6} \mathrm{H}_{6}$ is clearly incompatible with the observations.

The $\mathrm{C}_{6} \mathrm{H}_{6}$ column densities (or upper limit values) integrated in the 3 to 0.2 mbar pressure range are summarized in Table 3 . Based on these results, we conclude that benzene is slightly 

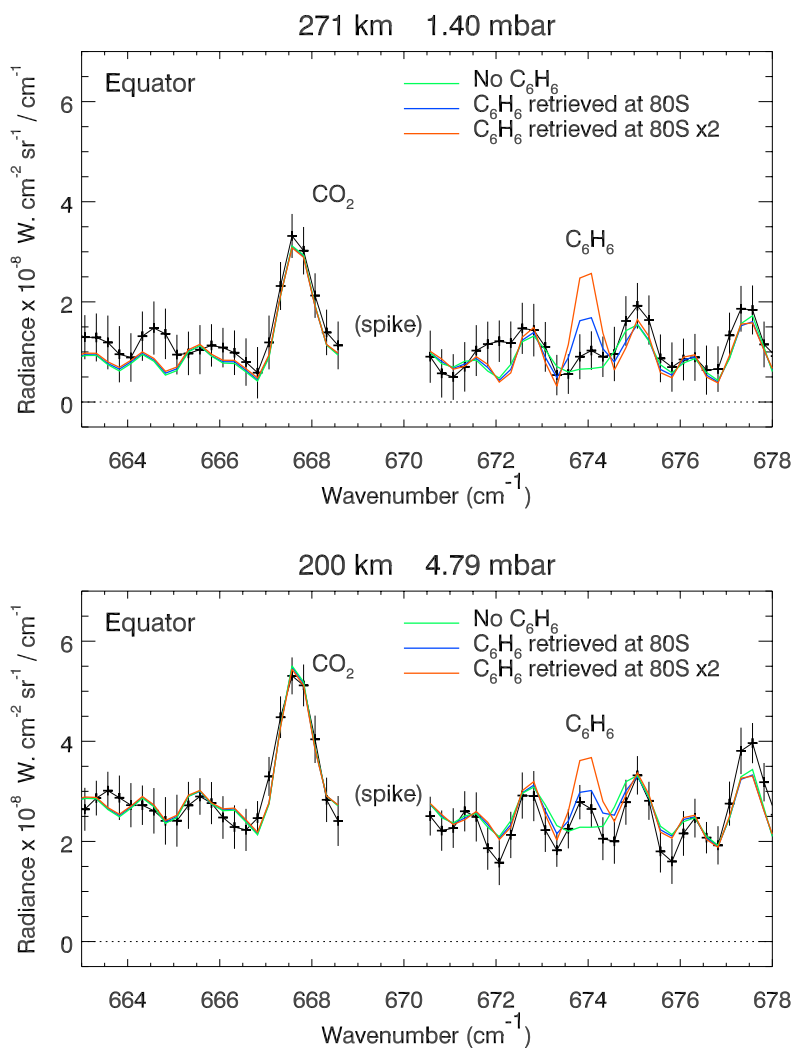

Fig. 6. Same as Fig. 5, for the equatorial dataset.

Table 3. Benzene column densities $\left(\mathrm{cm}^{-2}\right)$ integrated in the 3-0.2 mbar pressure range retrieved from CIRS, compared to the Moses \& Greathouse (2005) photochemical model.

\begin{tabular}{cccc}
\hline \hline Latitude & CIRS $^{a}$ & CIRS $^{b}$ & Model \\
\hline $40^{\circ} \mathrm{N}$ & $2.3 \pm 0.5 \times 10^{13}$ & $3.3 \pm 0.6 \times 10^{13}$ & $1.6 \times 10^{14}$ \\
$0^{\circ}$ & $<3.8 \times 10^{13}$ & $<3.9 \times 10^{13}$ & $3.8 \times 10^{15}$ \\
$35^{\circ} \mathrm{S}$ & $<2.9 \times 10^{13}$ & $<3.7 \times 10^{13}$ & $1.1 \times 10^{15}$ \\
$80^{\circ} \mathrm{S}$ & $4.1 \pm 1.0 \times 10^{13}$ & $4.1 \pm 0.9 \times 10^{13}$ & $8.2 \times 10^{13}$ \\
\hline
\end{tabular}

Notes. ${ }^{(a)}$ Prior profile equal to $\left[\mathrm{C}_{2} \mathrm{H}_{2}\right] / 10000 .{ }^{(b)}$ Prior profile from Moses \& Greathouse (2005).

enhanced at $80^{\circ} \mathrm{S}$ compared to $40^{\circ} \mathrm{N}$, and that the equatorial region is not significantly enriched in $\mathrm{C}_{6} \mathrm{H}_{6}$ compared to $80^{\circ} \mathrm{S}$.

\subsection{Retrieval of aerosol opacity profiles}

At $80^{\circ} \mathrm{S}$, after the retrieval of the temperature and hydrocarbon profiles, our best-fit spectra still do not match the observations in several spectral regions in particular around $700 \mathrm{~cm}^{-1}$, $750 \mathrm{~cm}^{-1}, 780 \mathrm{~cm}^{-1}$ and $1380-1480 \mathrm{~cm}^{-1}$ (Figs. 7 and 8). These residual signatures are visible in spectra at four different tangent heights, corresponding to tangent pressure levels of 17.8, 3.4, 0.9 , and 0.2 mbar. One striking example is the fit to the propane band, centred at $748 \mathrm{~cm}^{-1}$ : without haze opacity, a rather large amount of $\mathrm{C}_{3} \mathrm{H}_{8}$ is needed to match the radiance level in the propane wings at 740 and $755 \mathrm{~cm}^{-1}$; however, the corresponding synthetic spectra overestimate the radiance in the core of the propane band. A model with less propane would match the radiance in the core of the band, but would underestimate the overall radiance. An extra source of opacity is thus needed to correctly reproduce the observed spectra.
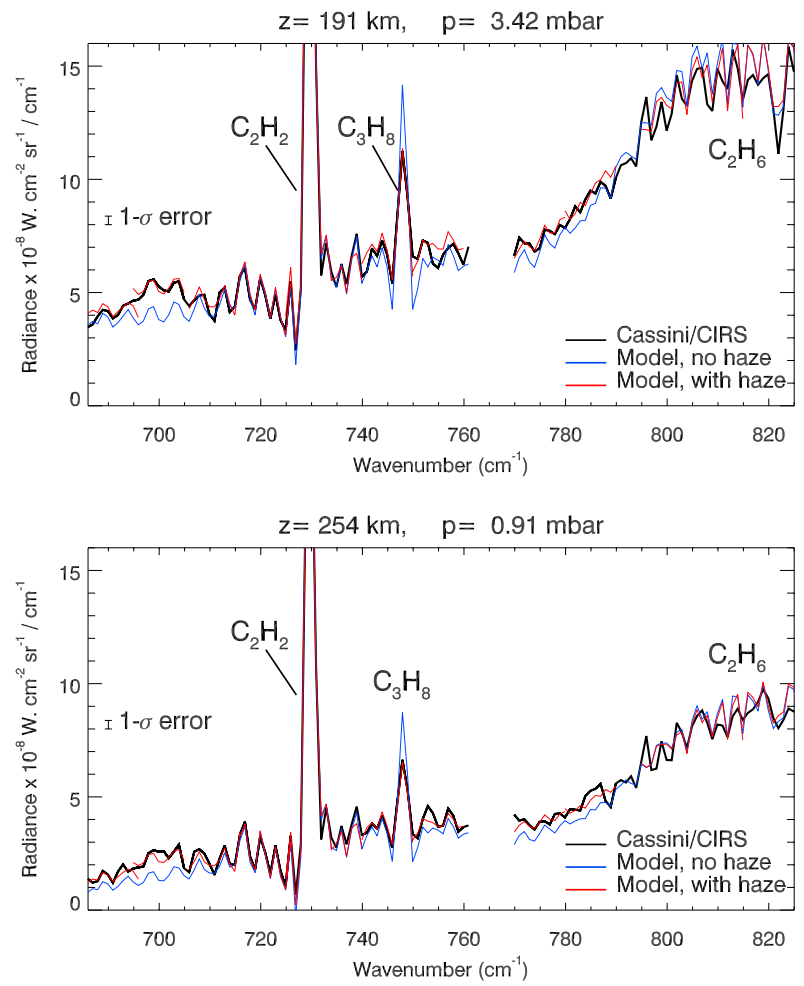

Fig. 7. Cassini/CIRS spectra acquired at $80^{\circ} \mathrm{S}$ at two different tangent heights (in black) compared to best-fit spectra, obtained by retrieving haze opacity profiles (in red) or assuming no haze opacity (in blue). A typical $1 \sigma$ measurement error due to noise is indicated.

After checking that no other minor species could significantly contribute to the emitted radiance in these regions, we implemented in the model the possibility of also retrieving an aerosol opacity profile. The radiative transfer model and optimal estimation retrieval method we use follow exactly that of Guerlet et al. (2009), except that we now take into account a grey aerosol opacity. Continuous aerosol opacity profiles (and not a scaling of a prior profile) are retrieved in small, independent, spectral regions (10 to $20 \mathrm{~cm}^{-1}$ wide) to constrain the spectral dependency of the haze opacity. Different prior profiles are tested, varying the aerosol scale height and opacity. Regarding the spectral region beyond $1370 \mathrm{~cm}^{-1}$ (which was not considered in Guerlet et al. 2009), in addition to methane, the radiative transfer model takes into account opacity from the $v_{6}$ and $v_{8} \mathrm{C}_{2} \mathrm{H}_{6}$ bands centred at 1378 and $1468 \mathrm{~cm}^{-1}$ (linelist from di Lauro et al. 2012), and from the $v_{18} \mathrm{C}_{3} \mathrm{H}_{8}$ band centred at $1376 \mathrm{~cm}^{-1}$ (Flaud et al. 2001).

One difficulty in this analysis lies in the potential degeneracy between the contribution of hazes and hydrocarbons to the opacity. However, this problem can be addressed as the spectral dependency of the haze opacity has no reason to follow that of the hydrocarbons. In addition, CIRS spectral resolution is high enough to resolve, if not individual lines, regions of higher and lower opacities within a given hydrocarbon band, which diversifies the information content of the spectra. Our methodology is to proceed in several steps. First, in spectral regions of strong gaseous opacity, the mixing ratio profile of the dominant trace species is retrieved simultaneously with a haze opacity profile: we successively retrieve $\mathrm{C}_{2} \mathrm{H}_{6}$ in the region $810-830 \mathrm{~cm}^{-1}$, then $\mathrm{C}_{2} \mathrm{H}_{2}$ in the region $725-740 \mathrm{~cm}^{-1}$, then $\mathrm{C}_{3} \mathrm{H}_{8}$ in the region $740-755 \mathrm{~cm}^{-1}$. This approach yields satisfactory fits to the data, both in regions of continuum or core hydrocarbon absorption (Fig. 7). A consequence of this new analysis of the $80^{\circ} \mathrm{S}$ dataset 


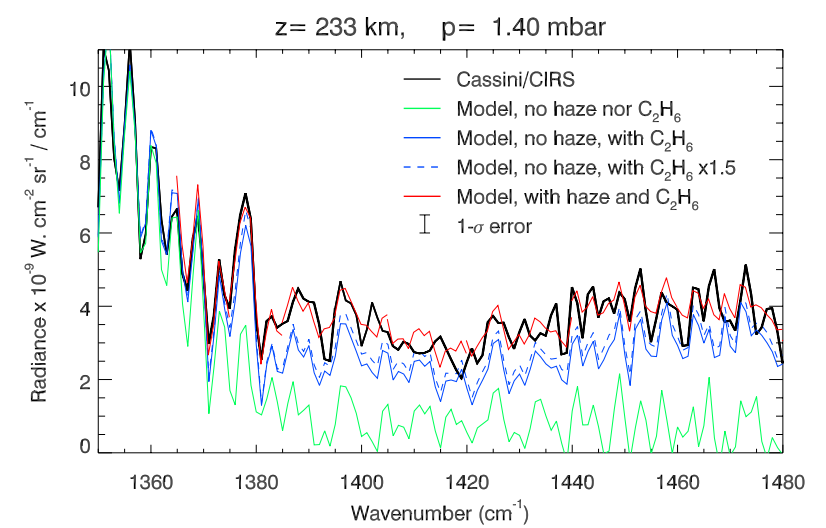

Fig. 8. Cassini/CIRS spectrum acquired at $80^{\circ} \mathrm{S}$ (in black) compared to best-fit spectra, obtained by retrieving haze opacity profiles (in red) or assuming no haze opacity (in blue). A synthetic spectrum without haze or $\mathrm{C}_{2} \mathrm{H}_{6}$ is also shown in green. A typical $1 \sigma$ measurement error due to noise is indicated.

is that the inferred $\mathrm{C}_{2} \mathrm{H}_{2}$ and $\mathrm{C}_{3} \mathrm{H}_{8}$ mixing ratios are smaller (by $20 \%$ and $50 \%$, respectively) than those retrieved without aerosol opacity in Guerlet et al. (2009). We emphasize that the results of Guerlet et al. (2009) at all other latitudes are not affected, and that their conclusions about the meridional variations of hydrocarbons still hold true.

Secondly, once the hydrocarbon profiles are constrained, their abundances are fixed and aerosol opacity profiles alone are retrieved in spectral regions of small or moderate gaseous opacities (e.g. $685-695 \mathrm{~cm}^{-1}, 695-705 \mathrm{~cm}^{-1}$, etc.). For each spectral bin, we check that the fit to the data is improved when aerosol opacity is included, otherwise the retrieved aerosol opacity profile is discarded.

The region $1360-1480 \mathrm{~cm}^{-1}$ has to be treated with caution. The haze retrieval can be hampered by uncertainties on the $\mathrm{C}_{2} \mathrm{H}_{6}$ abundance $(\sim 20-30 \%)$ or by spectroscopic errors on the line intensities ( $\sim 20 \%$, di Lauro et al. (2012)). In this spectral region, $\mathrm{C}_{2} \mathrm{H}_{6}$ is not retrieved but rather is set to the abundance profile determined from the strong $v_{9}$ band at $820 \mathrm{~cm}^{-1}$, and spectroscopic inconsistencies between the $v_{6}, v_{8}$, and $v_{9}$ bands could induce systematic errors. We thus evaluate the sensitivity of the haze retrieval to the $\mathrm{C}_{2} \mathrm{H}_{6}$ abundance in the region $1360-1480 \mathrm{~cm}^{-1}$. Figure 8 shows that synthetic spectra assuming no haze but $50 \%$ more $\mathrm{C}_{2} \mathrm{H}_{6}$ than is retrieved from the $v_{9}$ band would still not match the observed radiance. More generally, we find that if haze opacity is omitted, a $\mathrm{C}_{2} \mathrm{H}_{6}$ enhancement of a factor of 3 would be needed to match the observations, which is well above the $\mathrm{C}_{2} \mathrm{H}_{6}$ error bars and spectroscopic errors. Even so, we find that the region $1380-1390 \mathrm{~cm}^{-1}$ has a very low sensitivity to the amount of $\mathrm{C}_{2} \mathrm{H}_{6}$ (see Fig. 8), which makes the detection of this aerosol feature robust. In parallel, we evaluate the error on the retrieved aerosol opacity related to aliasing effects in CIRS spectra. Aliasing causes part of the spectra beyond $1529 \mathrm{~cm}^{-1}$ (the end of CIRS FP4 bandpass) to fold back at smaller wavenumbers, with some weighting factor, and would tend to overestimate the observed radiance. These effects depend with wavenumber on the weighting of the numerical filter and gradually increase beyond $1433 \mathrm{~cm}^{-1}$. They are estimated to be of the order of $5 \%$ of the observed radiance at $1440 \mathrm{~cm}^{-1}, 10 \%$ at $1460 \mathrm{~cm}^{-1}$, and $15 \%$ at $1480 \mathrm{~cm}^{-1}$. To fully take into account aliasing effects, one would have to compute synthetic spectra up to $1700 \mathrm{~cm}^{-1}$, then fold it back with appropriate weighting. However, the issue remains that we do not know the haze spectrum in that spectral region, hence we cannot

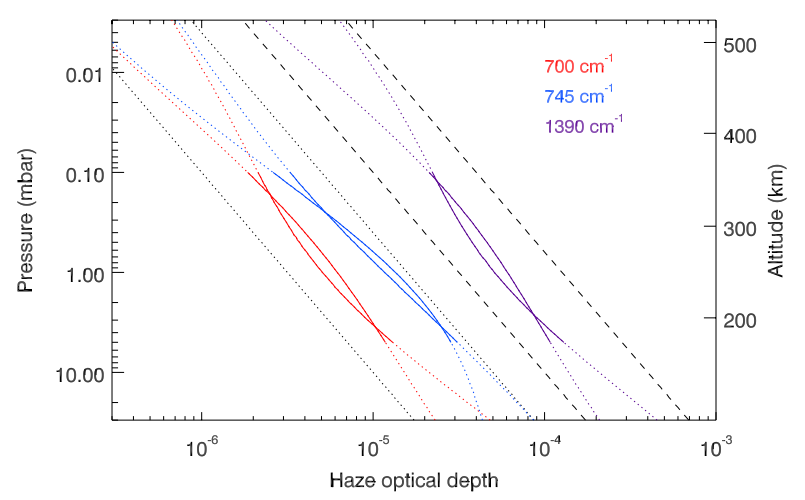

Fig. 9. Examples of retrieved haze opacity profiles at $80^{\circ} \mathrm{S}$ for the spectral ranges $695-705 \mathrm{~cm}^{-1}$ (red lines), $740-750 \mathrm{~cm}^{-1}$ (blue lines), and $1385-1395 \mathrm{~cm}^{-1}$ (purple lines). For each spectral range, retrieved profiles starting from two different prior profiles (dotted and dashed black lines) are shown. In this example, the prior profiles are proportional to $p^{0.5}$, with $p$ the pressure.

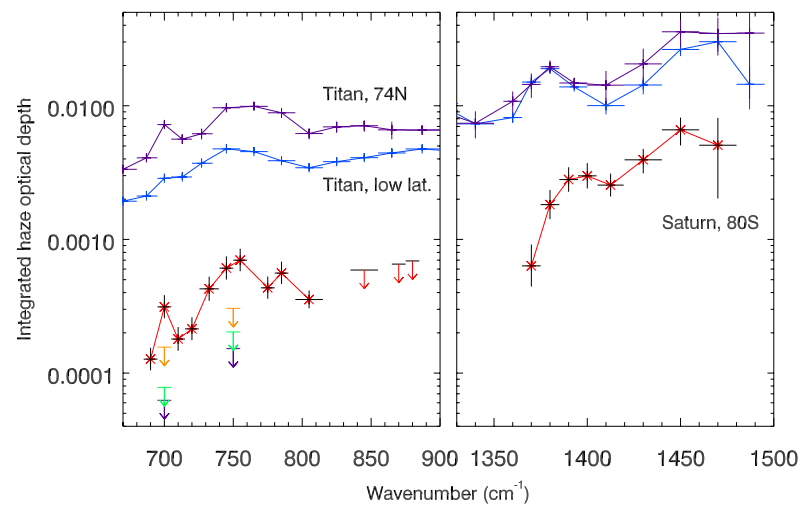

Fig. 10. Spectrum of the haze opacity integrated between 3 and $0.1 \mathrm{mbar}$ at $80^{\circ} \mathrm{S}$ (Saturn, in red). Upper limits at 700 and $750 \mathrm{~cm}^{-1}$ are shown for $40^{\circ} \mathrm{N}, 0^{\circ}$, and $35^{\circ} \mathrm{S}$ (purple, green, and orange arrows). For comparison, Titan's haze opacity integrated between 1.7 and 0.7 mbar, derived at $74^{\circ} \mathrm{N}$ and at low latitudes, are shown as purple and blue lines (Vinatier et al. 2012).

compute synthetic spectra with a high confidence level. Instead, in order to evaluate these aliasing effects on the haze retrievals, we perform test retrievals assuming lower radiances (by 5, 10, or $15 \%$ depending on wavenumber) than the calibrated ones in the range $1440-1480 \mathrm{~cm}^{-1}$. The more extreme case corresponds to an error of $\pm 50 \%$ on the integrated haze opacity.

Using this method, we constrain haze opacity profiles between $\sim 8$ mbar and 0.1 mbar in each spectral bin. Examples of retrieved aerosol opacity profiles are shown in Fig. 9. We find that the aerosol scale height is about twice the pressure scale height, which suggests that these aerosols are produced at high altitudes and sediment in the lower stratosphere, where they accumulate. The haze integrated opacity and its spectral dependency is shown in Fig. 10. Since a grey aerosol opacity is retrieved within spectral bins $10-20 \mathrm{~cm}^{-1}$ wide, the spectral shape of the aerosol signatures are only crudely resolved. For this reason, some discrepancies in the best-fit synthetic spectra can appear from one bin to another, for instance at $695 \mathrm{~cm}^{-1}$, as the aerosol opacity increases sharply between 690 and $700 \mathrm{~cm}^{-1}$ (see Fig. 7). In spite of the limited spectral resolution, the haze integrated opacity exhibits distinct spectral bands centred at 700 , $750,780,1390$, and $1450 \mathrm{~cm}^{-1}$. Interestingly, similar spectral 
features are also observed in Titan's stratosphere (Vinatier et al. 2012) and will be discussed in Sect. 4.2.

Finally, we derive upper limits on the haze opacity at $40^{\circ} \mathrm{N}$, $35^{\circ} \mathrm{S}$, and at the equator. We focus on deriving upper limits for the main aerosol signatures seen at 700 and $750 \mathrm{~cm}^{-1}$ by computing synthetic spectra with a haze opacity profile fixed to that retrieved at $80^{\circ} \mathrm{S}$ multiplied by a scaling factor. The upper limits we derive correspond to integrated opacities 2 to 5 times smaller than that at $80^{\circ} \mathrm{S}$ (see Fig. 10). Unfortunately, the same exercise in the region $1360-1480 \mathrm{~cm}^{-1}$ did not yield restrictive upper limits because the Planck function (hence the sensitivity to aerosol opacity) is smaller in this spectral range. The lower atmospheric temperatures at $40^{\circ} \mathrm{N}, 35^{\circ} \mathrm{S}$, and at the equator imply that the observed spectra are compatible with a no-haze scenario or with the same haze opacity as at $80^{\circ} \mathrm{S}$.

\section{Implications for Saturn's polar chemistry}

\subsection{Benzene chemistry}

The benzene partial column abundances derived from CIRS (or their upper limits) are in strong disagreement with the predictions of the photochemical model of Moses \& Greathouse (2005, which only includes neutral chemistry), both in terms of absolute values and meridional trends. Indeed, the equatorial and midlatitude benzene abundances in Moses \& Greathouse (2005) are too high by one to two orders of magnitude compared to the values or upper limits derived from CIRS (and ISO) measurements. At these latitudes, the impact of ion chemistry should be minor, implying that neutral benzene chemistry (its production/loss pathways and/or reaction constants) has to be revisited.

Our results show that at $80^{\circ} \mathrm{S}$ on Saturn, benzene is comparable to or even slightly higher than its equatorial and mid-latitude values. This contrasts with the Moses \& Greathouse (2005) photochemical model, which predicts a maximum benzene abundance at the equator, and about 50 times lower at $80^{\circ} \mathrm{S}$ (see Table 3). Two hypotheses could explain this data-model mismatch: i) the $\mathrm{C}_{6} \mathrm{H}_{6}$ meridional trend predicted by the Moses \& Greathouse (2005) 1D photochemical scheme is valid, but atmospheric advection (subsidence over the auroral regions and/or meridional transport) efficiently enriches the middle stratosphere in benzene; or ii) ion chemistry significantly increases the benzene production in the auroral regions. The dynamical scenario (without ion chemistry) is not favoured for two reasons. First, in the Moses \& Greathouse (2005) neutral photochemical model, the benzene production is highest around the 1 mbar pressure level, creating a local maximum in benzene abundance (see Fig. 4). Consequently, subsidence above the 1 mbar pressure level would not efficiently enrich the low and middle stratosphere in the region probed by CIRS (3-0.1 mbar). Secondly, meridional transport to the polar regions would not be efficient either, as benzene has a chemical loss time scale of 2-3 Earth years in the 1 mbar region, much shorter than the presumed timescale of meridional transport (100-700 years, Howett et al. 2007; Guerlet et al. 2009; Hesman et al. 2009). Hence, benzene is expected to be more rapidly destructed before it can be transported to the polar regions.

Regarding ion chemistry, Wong et al. (2000) showed that ionization by energetic auroral electrons is essential to benzene formation in Jupiter's upper atmosphere: in their model, if the ion-related reactions are omitted, $\mathrm{C}_{6} \mathrm{H}_{6}$ in the polar stratosphere is reduced by a factor of 550. Saturn's auroral power is about ten times weaker than Jupiter's, however, and its role on benzene formation had not been previously studied.
Hence, we favour the hypothesis that ion chemistry plays an important role in producing benzene in Saturn's polar stratosphere, similar to the production pathway suggested for Jupiter's stratosphere. Under this assumption, the benzene production region could extend to higher altitudes (as on Titan - Vuitton et al. 2008; and Jupiter - Wong et al. 2003) and consequently, subsidence could also play a part in the observed benzene enrichment. However, the role played by dynamics cannot be assessed without a better knowledge of the benzene production and loss rates. Our results thus strongly advocate for the inclusion of ion-related reactions in current Saturn chemical models.

\subsection{Aerosol composition and chemistry}

From the analysis of CIRS limb data, we report on the detection of several aerosol signatures in the thermal infrared present at $80^{\circ} \mathrm{S}$, but not detected at lower latitudes. As this coincides with the location of aurorae $\left(75^{\circ}\right.$ on average), this detection supports the link between the precipitation of energetic electrons and the production of aerosols, as suggested by previous Voyager and HST observations (West et al. 1983; Pryor \& Hord 1991; Karkoschka \& Tomasko 1993; Gérard et al. 1995).

These signatures exhibit striking similarities with those observed in Titan's lower stratosphere, with emission bands at $700,750-780,1380-1400$, and 1440-1480 $\mathrm{cm}^{-1}$. Vinatier et al. (2012) have assigned these bands to vibration modes of aliphatic and aromatic hydrocarbons (i.e. containing or not an aromaticring), with only a marginal contribution by amine vibration modes $(\mathrm{N}-\mathrm{N}$ or $\mathrm{N}-\mathrm{H})$. As the nitrogen content is very low in Saturn's stratosphere, we expect Saturn's aerosols to comprise mainly $\mathrm{C}$ and $\mathrm{H}$ atoms. Hence, the fact that these particular aerosol signatures are observed on both atmospheres strengthens the Vinatier et al. (2012) interpretation that they can be attributed to $-\mathrm{CH},-\mathrm{CH}_{2}$ and $-\mathrm{CH}_{3}$ deformation modes.

Whether these hydrocarbons build up as multi-ring compounds, long chains, or a disordered amorphous phase remains an open question. For Jupiter's polar atmosphere, Wong et al. (2000) propose a formation scheme in which (starting from benzene) successive hydrogen abstraction followed by acetylene addition leads to the formation of polycyclic aromatic hydrocarbons (PAH): naphthalene (2 rings, noted $A_{2}$ ), phenanthrene $\left(A_{3}\right)$, pyrene $\left(A_{4}\right)$, etc. An exploration of infrared spectroscopic databases (e.g. Silverstein 2005) reveals that the two signatures seen at 700 and $750 \mathrm{~cm}^{-1}$ are typical of out-of-plane $\mathrm{C}-\mathrm{H}$ vibrations in monosubstituted benzene molecules such as phenyl acetylene, a precursor to the formation of naphthalene in the Wong et al. (2000) scenario. In addition, naphthalene possesses a strong vibration band at $783 \mathrm{~cm}^{-1}$ and is thus a candidate for the observed aerosol signature centred at $780 \mathrm{~cm}^{-1}$. The signatures of $A_{3}$ (with bands at 735 and $815 \mathrm{~cm}^{-1}$ ) and $A_{4}$ $\left(850 \mathrm{~cm}^{-1}\right)$ are not detected in CIRS spectra, but they would be produced in smaller quantities than benzene or naphthalene (Wong et al. 2000). Hence, the combined observation of benzene and bands at 700,750, and $780 \mathrm{~cm}^{-1}$, which are typical of aromatics, is compatible with the Wong et al. (2000) PAH formation scenario.

On the other hand, the bands at $1380-1400 \mathrm{~cm}^{-1}$ and $1430-1480 \mathrm{~cm}^{-1}$ are typical of $-\mathrm{CH}_{2,3}$ vibration modes in aliphatic hydrocarbons, which suggests that both aromatic and non-aromatic $\mathrm{C}-\mathrm{H}$ bonds are present in polar aerosols. Based on the integrated aerosol opacity in the region $1430-1480 \mathrm{~cm}^{-1}$ and given the integrated cross-section of $-\mathrm{CH}_{2,3}$ in amorphous carbon at this wavenumber $\left(A=1.5 \pm 0.5 \times 10^{-18} \mathrm{~cm}\right.$ per $\mathrm{CH}_{2,3}$ group, from Dartois \& Muñoz-Caro 2007), we estimate 
the number of $-\mathrm{CH}_{2,3}$ groups to be $1.7 \pm 0.9 \times 10^{17} \mathrm{~cm}^{-2}$ in the considered partial column (3 to 0.1 mbar).

\subsection{Constraints on the aerosol mass loading}

Given the particle sizes and their optical properties, it is possible to derive an estimate of the aerosol partial column density and mass loading. Here we assume that aerosols are spherical particle aggregates with a radius $r$ of $0.1 \mu \mathrm{m}$ (consistent with Pérez-Hoyos et al. 2005). Using Mie scattering theory, we compute the haze extinction coefficient $Q_{\text {ext }}$ (how the aerosol optical properties are chosen is detailed in Sect. 5). The extinction coefficient is linked to the aerosol column density, $N$ through

$\tau_{\text {int }, \lambda}=\pi r^{2} Q_{\text {ext }, \lambda} N$.

Applying Eq. (1) with the retrieved integrated aerosol opacity $\tau_{\text {int }}$ yields an integrated column density $N$ of $3 \pm 0.8 \times 10^{9}$ particles $\mathrm{cm}^{-2}$ in the 3 to 0.1 mbar pressure range. Assuming a particule density of $1 \mathrm{~g} \mathrm{~cm}^{-3}$ (typically used for Jupiter and Titan, e.g. Zhang et al. 2013; Lavvas et al. 2011), this implies an aerosol mass loading of $1.3 \pm 0.3 \times 10^{-5} \mathrm{~g} \mathrm{~cm}^{-2}$ in the 3 to 0.1 mbar pressure range (or $3.7 \pm 0.9 \times 10^{-5} \mathrm{~g} \mathrm{~cm}^{-2}$ if we extrapolate our results down to the $20 \mathrm{mbar}$ pressure level). In comparison, Zhang et al. (2013) estimated the aerosol mass loading in Jupiter's polar stratosphere to be of the order of $10^{-4} \mathrm{~g} \mathrm{~cm}^{-2}$. Given that Saturn's auroral power is about ten times weaker than Jupiter's (Gérard \& Singh 1982), the fact that we derive an aerosol mass loading an order of magnitude less than on Jupiter hints towards a possibly similar formation processes.

Finally, the aerosol mass loading corresponds to a number of carbon atoms of $6 \pm 1.4 \times 10^{17} \mathrm{~cm}^{-2}$, about three times the previously-derived estimate of the number of aliphatic $-\mathrm{CH}_{2,3}$ groups. Hence, these figures are consistent with the assumption that aerosols comprise $\mathrm{C}$ atoms involved in their backbone structure, such as less IR active aliphatic $\mathrm{C}-\mathrm{C}$, olefinic $\mathrm{C}=\mathrm{C}$, and/or aromatic $\mathrm{C}=\mathrm{C}$ bonds, but also $\mathrm{IR}$ active aromatic $\mathrm{C}-\mathrm{H}$ bonds.

\section{Modelling the haze's radiative impact}

To evaluate the radiative impact of this polar haze on the thermal structure, we employ the seasonal radiative-convective model of Saturn's atmosphere described in Guerlet et al. (2014), in which we include an additional polar stratospheric haze layer. The input haze opacity vertical profile is constructed given the retrieved aerosol scale height (twice the pressure scale height), the integrated opacity, and the bottom pressure level of the haze layer. The last is not well constrained by observations and we evaluate two scenarios: one with a haze layer extending between 0 and 50 mbar, with an integrated haze opacity at $300 \mathrm{~nm}$ set to 0.6 (corresponding to the Karkoschka \& Tomasko 2005 scenario) and one concentrated in the $0-10$ mbar region, with an integrated opacity of 0.25 (the Pérez-Hoyos et al. 2005 scenario). Another similar and intermediate scenario, not considered here, is the one derived from the analysis of Cassini/ISS observations by Sánchez-Lavega et al. (2006), in which the haze layer extends from 1 to 30 mbar with an integrated opacity of 0.54 at $300 \mathrm{~nm}$.

Real and imaginary refractive indexes are taken from Karkoschka \& Tomasko (2005) for the UV and visible range, applying their Eq. (3) with $m_{i}=0.08$ at $300 \mathrm{~nm}$. For the thermal infrared, we start by using the Vinatier et al. (2012) refractive indexes, given the similarities between Saturn's and Titan's aerosol spectra at these wavenumbers. We then slightly adjust the imaginary part of the refractive index so that the modelled integrated

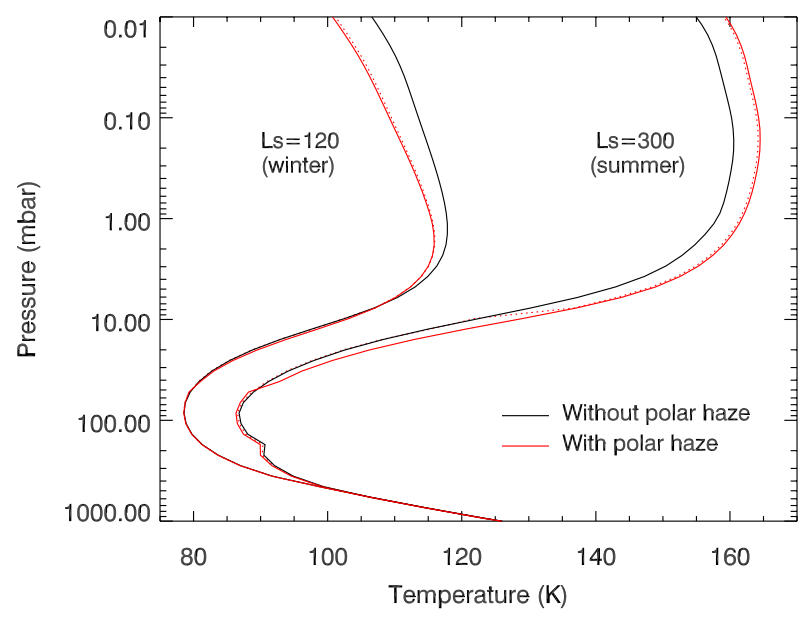

Fig. 11. Equilibrium temperature profiles simulated at $80^{\circ} \mathrm{S}$, with or without a stratospheric polar haze layer, for mid-summer $\left(\mathrm{Ls}=300^{\circ}\right)$ and mid-winter $\left(\mathrm{Ls}=120^{\circ}\right)$ conditions. Two scenarios are illustrated, with a haze layer extending down to 50 mbar (solid red line) or $10 \mathrm{mbar}$ (dotted red line).

aerosol opacities in the thermal infrared match that observed in this study. Refractive indexes in the visible and infrared part are summarized in Table 4.

Figure 11 presents equilibrium temperature profiles calculated at $80^{\circ} \mathrm{S}$ with or without a stratospheric polar haze layer, for summer and winter conditions. We find that this polar haze induces a net stratospheric heating during summer, reaching $+6 \mathrm{~K}$ at the 10 mbar pressure level, and a net stratospheric cooling during winter, reaching $-5 \mathrm{~K}$ at and above the 0.1 mbar pressure level. The two aerosol scenarios yield very similar results, only differing in the 10-50 mbar region where the Pérez-Hoyos et al. (2005) scenario is colder in summer in this region because it lacks aerosols. The properties of the haze (particle sizes and optical depth) are likely to vary with seasons, however, so that these figures are qualitative. In order to provide a range of plausible figures, we evaluated the sensitivity of the haze's radiative impact to different opacities: if the aerosol optical depth is two times smaller (resp. two times higher), the heating only amounts to $+4 \mathrm{~K}$ in summer (resp. $+8 \mathrm{~K}$ ) and the cooling to $-4 \mathrm{~K}$ in winter (resp. $-6.5 \mathrm{~K}$ ).

Even though the precise evaluation of the polar haze radiative impact would require complex seasonal radiative and chemical modelling, this warming effect is reminiscent of the observation of a warm stratospheric polar hood in mid-summer (Ls = $300^{\circ}$ ), where the temperature at 1 mbar sharply increases (by $\sim 8 \mathrm{~K}$ ) between 70 and $80^{\circ} \mathrm{S}$ (Orton \& Yanamandra-Fisher 2005; Fletcher et al. 2008). Our modelled temperature profiles suggest that auroral-induced aerosols confined in the polar hood could significantly contribute to this polar warming, although we cannot rule out that other processes, such as adiabatic heating due to subsidence, also play a part in the warming. This hypothesis has been suggested in previous studies (Orton \& Yanamandra-Fisher 2005; Sánchez-Lavega et al. 2006; Fletcher et al. 2008, 2015), but this is the first time that this effect is estimated.

\section{Summary and conclusions}

Using Cassini/CIRS limb observations, we derive the first measurement of the benzene column abundance in Saturn's auroral region, which is found to be comparable to or slightly higher than mid-latitude and equatorial regions. This is in contradiction 
Table 4. Imaginary part of the wavelength-dependent aerosol refractive index used to compute extinction coefficients.

\begin{tabular}{cc}
\hline \hline Wavelength $(\mu \mathrm{m})$ & $m_{i}$ \\
\hline 0.255 & $4.00 \times 10^{-1}$ \\
0.300 & $1.60 \times 10^{-1}$ \\
0.336 & $1.00 \times 10^{-1}$ \\
0.42 & $2.48 \times 10^{-2}$ \\
0.55 & $2.84 \times 10^{-3}$ \\
0.65 & $1.35 \times 10^{-3}$ \\
0.70 & $1.00 \times 10^{-3}$ \\
0.80 & $6.40 \times 10^{-4}$ \\
0.90 & $4.80 \times 10^{-4}$ \\
\hline 3.00 & $1.00 \times 10^{-3}$ \\
4.00 & $1.00 \times 10^{-3}$ \\
5.00 & $4.00 \times 10^{-3}$ \\
6.60 & $1.00 \times 10^{-2}$ \\
6.90 & $1.80 \times 10^{-1}$ \\
7.05 & $6.00 \times 10^{-2}$ \\
7.25 & $1.00 \times 10^{-1}$ \\
7.35 & $5.00 \times 10^{-2}$ \\
8.00 & $1.00 \times 10^{-2}$ \\
9.00 & $1.00 \times 10^{-2}$ \\
10.00 & $3.00 \times 10^{-2}$ \\
13.33 & $3.25 \times 10^{-2}$ \\
13.90 & $0.85 \times 10^{-2}$ \\
14.30 & $1.75 \times 10^{-2}$ \\
15.00 & $0.70 \times 10^{-2}$ \\
20.00 & $0.70 \times 10^{-2}$ \\
25.00 & $2.00 \times 10^{-2}$ \\
30.00 & $3.00 \times 10^{-2}$ \\
35.00 & $3.00 \times 10^{-2}$ \\
40.00 & $3.00 \times 10^{-2}$ \\
\hline &
\end{tabular}

with meridional trends predicted from the Moses \& Greathouse (2005) photochemical model, and hence advocates for the inclusion of ion chemistry in Saturn's chemical models. Including the ion chemistry is also expected to affect the abundance of $\mathrm{C}_{2}$ hydrocarbons in the polar regions and would provide a new light on the interpretation of the observed meridional distribution of these species. In parallel, we find that the benzene abundances predicted by Moses \& Greathouse (2005) are generally overestimated, which raises the need for an update of the benzene chemistry (production/loss pathways and/or reaction constants).

We also present the first characterization of Saturn's polar stratospheric aerosols in the thermal infrared. Their spectral properties show emission bands in the region $700-800 \mathrm{~cm}^{-1}$ and $1380-1480 \mathrm{~cm}^{-1}$ that are characteristic of vibration modes in aromatic and aliphatic hydrocarbons, presenting striking similarities with Titan's stratospheric aerosols observations. The aerosol mass loading in Saturn's polar stratosphere is estimated to be one order of magnitude lower than on Jupiter, consistent with their respective auroral intensities. Our results suggest that the aerosol scenario formation proposed by Wong et al. (2000) for Jupiter's polar stratosphere (producing PAH) could also be valid for Saturn's stratosphere, but that aliphatic bonds are also important in Saturn's aerosols. Complementary observations in the $3 \mu \mathrm{m}$ region (for instance with Cassini/VIMS) could help further characterize the polar haze; aromatics possess vibration bands centred at $3050 \mathrm{~cm}^{-1}(3.28 \mu \mathrm{m})$, while aliphatic $\mathrm{C}-\mathrm{H}$ stretch bands exhibit distinct signatures centred at $2950 \mathrm{~cm}^{-1}(3.4 \mu \mathrm{m})$.

Finally, Saturn's polar aerosols are estimated to have a significant radiative impact on the stratospheric temperature and could be partly responsible for the warm polar hood observed in the lower stratosphere during summer at latitudes polewards of $75^{\circ} \mathrm{S}$ (Fletcher et al. 2008, 2015). Future measurements of the temperature and haze opacities in the polar regions, in particular the monitoring of the formation - or not - of a northern counterpart of the south polar hood, will be extremely valuable for the study of the chemistry and radiative budget in Saturn's polar stratosphere.

Acknowledgements. S. Guerlet acknowledges funding by the French ANR under grant agreement ANR-12-PDOC-0013. T. Fouchet acknowledges funding by the Institut Universitaire de France. We thank Julie Moses and Jim Friedson for discussions on aerosol formation and Conor Nixon for discussions on aliasing effects in CIRS spectra, and an anonymous referee whose comments helped to improve the manuscript. We also thank the CIRS operation and calibration team for their hard work.

\section{References}

Bézard, B., Drossart, P., Encrenaz, T., \& Feuchtgruber, H. 2001, Icarus, 154, 492 Conrath, B. J., \& Gautier, D. 2000, Icarus, 144, 124

Dartois, E., \& Muñoz-Caro, G. M. 2007, A\&A, 476, 1235

di Lauro, C., Lattanzi, F., Brown, L. R., et al. 2012, Planet. Space Sci., 60, 93

Flasar, F. M., Kunde, V. G., Abbas, M. M., et al. 2004, Space Sci. Rev., 115, 169

Flasar, F. M., Achterberg, R. K., Conrath, B. J., et al. 2005, Science, 307, 1247

Flaud, J.-M., Lafferty, W. J., \& Herman, M. 2001, J. Chem. Phys., 114, 9361

Fletcher, L. N., Irwin, P. G. J., Orton, G. S., et al. 2008, Science, 319, 79

Fletcher, L. N., Hesman, B. E., Achterberg, R. K., et al. 2012, Icarus, 221, 560

Fletcher, L. N., Irwin, P. G. J., Sinclair, J. A., et al. 2015, Icarus, 250, 131

Fouchet, T., Guerlet, S., Strobel, D. F., et al. 2008, Nature, 453, 200

Friedson, A. J., Wong, A.-S., \& Yung, Y. L. 2002, Icarus, 158, 389

Gérard, J. C., \& Singh, V. 1982, J. Geophys. Res., 87, 4525

Gérard, J. C., Dols, V., Grodent, D., et al. 1995, Geophys. Res. Lett., 22, 2685

Guerlet, S., Fouchet, T., Bézard, B., Simon-Miller, A. A., \& Michael Flasar, F. 2009, Icarus, 203, 214

Guerlet, S., Fouchet, T., Bézard, B., et al. 2010, Icarus, 209, 682

Guerlet, S., Spiga, A., Sylvestre, M., et al. 2014, Icarus, 238, 110

Hesman, B. E., Jennings, D. E., Sada, P. V., et al. 2009, Icarus, 202, 249

Howett, C. J. A., Irwin, P. G. J., Teanby, N. A., et al. 2007, Icarus, 190, 556

Karkoschka, E., \& Tomasko, M. G. 1993, Icarus, 106, 428

Karkoschka, E., \& Tomasko, M. 2005, Icarus, 179, 195

Lavvas, P., Sander, M., Kraft, M., \& Imanaka, H. 2011, ApJ, 728, 80

Moses, J. I., \& Greathouse, T. K. 2005, J. Geophys. Res., 110, 9007

Orton, G. S., \& Yanamandra-Fisher, P. A. 2005, Science, 307, 696

Pérez-Hoyos, S., Sánchez-Lavega, A., French, R. G., \& Rojas, J. F. 2005, Icarus, 176,155

Pryor, W. R., \& Hord, C. W. 1991, Icarus, 91, 161

Silverstein, R. M., Webster, F. X., D. K. 2005, Spectrometric Identification of Organic Compounds (John Wiley \& Sons)

Sánchez-Lavega, A., Hueso, R., Pérez-Hoyos, S., \& Rojas, J. F. 2006, Icarus, 184,524

Vinatier, S., Rannou, P., Anderson, C. M., et al. 2012, Icarus, 219, 5

Vuitton, V., Yelle, R. V., \& Cui, J. 2008, J. Geophys. Res. (Planets), 113, 5007

West, R. A., Hord, C. W., Simmons, K. E., et al. 1983, Adv. Space Res., 3, 45

Wong, A.-S., Lee, A. Y. T., Yung, Y. L., \& Ajello, J. M. 2000, ApJ, 534, L215

Wong, A.-S., Yung, Y. L., \& Friedson, A. J. 2003, Geophys. Res. Lett., 30, 1447

Zhang, X., West, R. A., Banfield, D., \& Yung, Y. L. 2013, Icarus, 226, 159 